Article

\title{
The Exact Evaluation of Some New Lattice Sums
}

\section{J. Zucker}

Physics Department, King's College, Strand, London WC2R 2LS, UK; jzucker@btinternet.com

Received: 14 November 2017; Accepted: 6 December 2017; Published: 11 December 2017

Abstract: New $q$-series in the spirit of Jacobi have been found in a publication first published in 1884 written in Russian and translated into English in 1928. This work was found by chance and appears to be almost totally unknown. From these entirely new $q$-series, fresh lattice sums have been discovered and are presented here.

Keywords: Jacobian $q$-series; closed form lattice sums

\section{Introduction. A Short History of Lattice Sums}

The Jacobian $\theta$-functions

$$
\begin{gathered}
\theta_{2}=\theta_{2}(q)=\sum_{-\infty}^{\infty} q^{(m-1 / 2)^{2}}=2 q^{1 / 4}\left(1+q^{2}+q^{6} \ldots+q^{n(n+1)} \ldots,\right. \\
\theta_{3}=\theta_{3}(q)=\sum_{-\infty}^{\infty} q^{m^{2}}=1+2 q+2 q^{4}+2 q^{9}+2 q^{16} \ldots, \\
\theta_{4}=\theta_{4}(q)=\sum_{-\infty}^{\infty}(-1)^{m} q^{m^{2}}=1-2 q+2 q^{4}-2 q^{9}+2 q^{16} \ldots
\end{gathered}
$$

enjoy many surprising relations amongst themselves. Two which are of importance here are

$$
2 \theta_{3}\left(q^{4}\right)=\theta_{3}+\theta_{4} \quad \text { and } \quad 2 \theta_{3}^{2}\left(q^{2}\right)=\theta_{3}^{2}+\theta_{4}^{2} .
$$

They also have the remarkable property that many even powers and products of their representations as infinite series may be reduced to a single series. Thus, consider the following typical example.

$$
\theta_{3}^{2}=\sum_{-\infty}^{\infty} \sum_{-\infty}^{\infty} q^{m^{2}+n^{2}}=1+4 \sum_{m=1}^{\infty} \frac{4 q^{m}}{1+q^{2 m}}
$$

a result found in Jacobi [1]. We can further expand the RHS of (5) to give

$$
\sum_{-\infty}^{\infty} \sum_{-\infty}^{\infty} q^{m^{2}+n^{2}}=1+4 \sum_{m=1}^{\infty}\left(q^{m}-q^{3 m}+q^{5 m} \ldots\right)=1+4 \sum_{n=0}^{\infty} \sum_{m=1}^{\infty}\left[q^{(4 n+1) m}-q^{(4 n+3) m}\right]
$$

This allows us to say something about the quadratic form $m^{2}+n^{2}$, namely that the number of solutions of the diophantine equation $m^{2}+n^{2}=N$ is equal to four times the difference between the number of divisors of $N$ of the form $4 n+1$ and the number of divisors of $N$ of the form $4 n+3$. It was to find arithmetic properties for other quadratic forms that Jacobi made use of relations such as (6).

Surprisingly, for such a brillant algorist he did not make use of the fact that summing over any function of the given quadratic form was equivalent to summing over its transformed representation. Hence, in the example given here 


$$
\sum^{\prime} f\left(m^{2}+n^{2}\right)=4 \sum_{n=0}^{\infty} \sum_{m=1}^{\infty}[f[(4 n+1) m]-f[(4 n+3) m])
$$

In the above, $\Sigma^{\prime}$ implies summation over both $m$ and $n$ from $-\infty$ to $\infty$, excluding the case when $m, n$ are simultaneously zero. This removes the " 1 " in front of Equations (2) and (3). Thus, consider

$$
\sum^{\prime} \frac{1}{\left(m^{2}+n^{2}\right)^{s}}=4 \sum_{n=0}^{\infty} \sum_{m=1}^{\infty}\left[\frac{1}{(4 n+1)^{s} m^{s}}-\frac{1}{(4 n+3)^{s} m^{s}}\right]
$$

The sums over $m$ and $n$ on the RHS of (6) are completely independent of one another. We have

$$
\sum_{m=1}^{\infty} \frac{1}{m^{s}}=\zeta(s) \text { and } \sum_{n=0}^{\infty}\left[\frac{1}{(4 n+1)^{s}}-\frac{1}{(4 n+3)^{s}}\right]=L_{-4}(s)=\beta(s) .
$$

Here $\beta(s)$ is a well-known Dirichlet series of period 4. So, we now have the

$$
\sum^{\prime} \frac{1}{\left(m^{2}+n^{2}\right)^{s}}=4 \zeta(s) \beta(s)
$$

This result has usually been ascribed to Hardy [2] in 1919. However, it was discovered over a hundred years after it had been published that this result first appeared in a paper by Lorenz [3] in 1871. One may ask why it took so long for this paper to become known. Ludvig Lorenz was a Danish theoretical physicist who had done some good work on electromagnetism. However, the paper in which he published (9) was unusual in that it was written in Danish and published in the first volume of a new Danish journal. It was thus highly unlikely to receive attention in the 19th century. A further oddity was that not a single reference was given. Nevertheless, it should be pointed out that Lorenz, in his paper, provided results similar to (9). Thus, $\sum^{\prime}\left(m^{2}+a n^{2}\right)^{-s}$ with $a=2,3,4, \sum^{\prime}\left(m^{2}+m n+n^{+}\right)^{-s}$, plus the much trickier $\Sigma^{\prime}\left(m^{2}-n^{2}\right)^{-s}$, where the summation in this case is over all values of $m, n$ for which $m^{2} \neq n^{2}$, were also given exactly. Specifically, he derived

$$
\begin{gathered}
\sum^{\prime} \frac{1}{\left(m^{2}+2 n^{2}\right)^{s}}=2 \zeta(s) L_{-8}(s) \\
L_{-8}(s)=\sum_{n=0}^{\infty}\left[\frac{1}{(8 n+1)^{s}}+\frac{1}{(8 n+3)^{s}}-\frac{1}{(8 n+5)^{s}}-\frac{1}{(8 n+7)^{s}}\right] \\
\sum^{\prime} \frac{1}{\left(m^{2}+3 n^{2}\right)^{s}}=2\left(1+2^{1-2 s}\right) \zeta(s) L_{-3}(s)
\end{gathered}
$$

where

$$
\begin{gathered}
L_{-3}(s)=\sum_{n=0}^{\infty}\left[\frac{1}{(3 n+1)^{s}}-\frac{1}{(3 n+2)^{s}}\right] \\
\sum^{\prime} \frac{1}{\left(m^{2}+4 n^{2}\right)^{s}}=2\left(1-2^{-s}+2^{1-2 s}\right) \zeta(s) L_{-4}(s), \\
\sum^{\prime} \frac{1}{\left(m^{2}+m n+n^{2}\right)^{s}}=6 \zeta(s) L_{-3}(s) \\
\sum_{\left.m^{2} \neq n^{2}\right)} \frac{1}{\left|m^{2}-n^{2}\right|^{s}}=4\left(1-2^{1-s}+2^{1-2 s}\right) \zeta^{2}(s)
\end{gathered}
$$

$L_{-3}$ and $L_{-8}$ are further examples of Dirichlet series.

Lorenz also indicated that the quadratic forrm $\left(m_{1}^{2}+m_{2}^{2}+m_{3}^{2}+m_{4}^{2}\right)$ and $\left(m_{1}^{2}+m_{2}^{2}+2 m_{3}^{2}+2 m_{4}^{2}\right)$ might succumb to a similar process. This, plus the fact that Lorenz derived (5) on his own from various 
$\theta$ relations, indicates that Lorenz had no access to Jacobi's thesis. For if he had, he would certainly have discovered (5) in Jacobi's thesis and also found that

$$
\theta_{3}^{4}=\sum_{-\infty}^{\infty} \sum_{-\infty}^{\infty} \sum_{-\infty}^{\infty} \sum_{-\infty}^{\infty} q^{m_{1}^{2}+m_{2}^{2}+m_{3}^{2}+m_{4}^{2}}=1+8 \sum_{m=0}^{\infty} \frac{\left.(m+1) q^{m+1}\right)}{1+(-q)^{m+1}} .
$$

from which it would have been simple to obtain

$$
\sum^{\prime} \frac{1}{\left(m_{1}^{2}+m_{2}^{2}+m_{3}^{2}+m_{4}^{2}\right)^{s}}=8\left(1-2^{2-2 s}\right) \zeta(s-1) \zeta(s) .
$$

It would appear that no work was done on the exact evaluations of multiple sums between Lorenz [3] and Hardy [2], and then nothing until 1973. However, the numerical evaluation of multiple sums became an important topic for physicists in the first half of the 20th century when X-ray studies showed that ionic salts consisted not of molecules but of interpenetrating lattices of ions. So, sums such as $\sum^{\prime}(-1)^{m+n+p} / \sqrt{m^{2}+n^{2}+p^{2}}$ became of interest. This sum is known as the Madelung [4] constant after the first person to give a reasonably accurate value of this very slowly conditionally converging sum. Additionally, when the rare gases crystallised into solids of a face-centred cubic lattice with the atoms interacting with $1 / r^{6}$ van der Waals interaction, sums such as $\Sigma^{\prime}\left[(m+n)^{2}+(n+p)^{2}+(p+m)^{2}\right]^{-6}$ were also required. These sums have become known as lattice sums, and this name has become attached to any summation involving inverse squares of binary products of indices. Additionally, the number of different indices has been termed the dimension of the sum. Thus, the Madelung constant is an example of a three-dimensional lattice sum. Then, in 1973 Glasser [5,6], in 1974 Zucker [7], and together in 1980 [8], reawakened interest in the exact evaluation of lattice sums and at last disinterred Lorenz. The representation of lattice sums by $\theta$ series was put on a more formal basis by use of the Mellin transform $M_{S}$ defined by

$$
M_{s}[f(t)]=\frac{1}{\Gamma(s)} \int_{0}^{\infty} t^{s-1} f(t) \mathrm{d} t
$$

Thus, for example,

$$
\sum^{\prime} \frac{1}{\left(m^{2}+n^{2}\right)^{s}}=M_{s} \sum^{\prime} \exp \left[-\left(m^{2}+n^{2}\right) t\right]=M_{s}\left[\theta_{3}^{2}\left(e^{-t}\right)-1\right]
$$

Since then, many 2,3,4,6, and 8-dimensional lattice sums and even a $5 \mathrm{~d}$ example have been evaluated exactly. Along with properties of Dirichlet $L$-series and many other aspects of lattice sums, these are given with numerous references in the book Lattice sums then and now [9].

Since this book was published, the writer received from his colleague M.L. Glasser a work found by chance of a translation of a Russian book by a P.S. Nazimov- "Applications of the theory of elliptic functions to the theory of numbers" [10], first published in Russian in Moscow in 1884. The translator, Arnold Ross, says nothing about Nazimov, merely that he received the suggestion to translate the book from L. E. Dickson and E.T. Bell, and finished it in 1928. Bell writes a preface himself describing how he received the book from James V. Ouspenky at a Toronto conference in 1924. Bell refers to Nazimov's remarkable book and that outside of Russia his work is hardly known. For this he blames Nazimov himself: "The author himself is largely responsible since the French abstract in the Journal de l'Ecole Polytechnique does scant justice to the book", but of Nazimov he says nothing at all. Even the omniscient Google has nothing to say about him except that he was a student of Nicolai Vasilievich Bugaev (1837-1903), a professor at Moscow University in 1867. Not even what his initials P.S. stand for can be found. It thus seems that Nazimov has been even more unfortunate than Lorenz in the exposure of his work. Another comparison with Lorenz is his lack of giving references. Although he quotes several authors he gives no sources apart from one-namely, "Theorie des Fonctions Elliptique". Briot and Bouget, (1875). Nazimov's book has no mention of lattice sums. Like Jacobi, he is solely 
concerned with arithmetical properties of certain quadratic forms. However, just as the $q$-series derived by Jacobi for products of $\theta$-functions can be transformed into a lattice sum, so may the results of Nazimov. His facility in providing these new $q$ relations is to be admired, and it is these new results with which the next section is concerned.

\section{Some Lattice Sums Derived from Nazimov's $\theta$ Function Relations}

It is first necessary to introduce some new relevant mathematical functions. The complete elliptic integral of the first kind is defined by

$$
K(k)=\int_{0}^{1} \frac{\mathrm{d} x}{\sqrt{\left(1-x^{2}\right)\left(1-k^{2} x^{2}\right)}}=\int_{0}^{\pi / 2} \frac{\mathrm{d} \theta}{\sqrt{1-k^{2} \sin ^{2} \theta}}
$$

where in (19) $k$ is known as the modulus. The complimentary modulus is defined as $k^{\prime}=\sqrt{1-k^{2}}$ and $K\left(k^{\prime}\right)$ is denoted by $K^{\prime}$. The relationships between $K$ and the $\theta$ functions are as follows,

$$
\frac{2 K}{\pi}=\theta_{3}^{2}, \quad \frac{2 K k}{\pi}=\theta_{2}^{2}, \quad \frac{2 k^{\prime} K}{\pi}=\theta_{4}^{2}, \quad \text { with } q=e^{-\pi \frac{K^{\prime}}{K}} .
$$

Additionally, in further results for lattice sums other Dirichlet series will be met, and we list these here. First, there are two well-known variations of the $\zeta$ function.

$$
\begin{aligned}
& \sum_{n=1}^{\infty} \frac{(-1)^{n-1}}{n^{s}}=\frac{1}{1^{s}}-\frac{1}{2^{s}}+\frac{1}{3^{s}}-\frac{1}{4^{s}} \ldots=\left(1-2^{1-s}\right) \zeta(s)=\eta(s) \\
& \sum_{n=0}^{\infty} \frac{1}{(2 n+1)^{s}}=\frac{1}{1^{s}}+\frac{1}{3^{s}}+\frac{1}{5^{s}}+\frac{1}{7^{s}} \ldots=\left(1-2^{-s}\right) \zeta(s)=\lambda(s)
\end{aligned}
$$

There is the following period 5 series

$$
L_{5}(s)=\sum_{n=0}^{\infty}\left[\frac{1}{(5 n+1)^{s}}-\frac{1}{(5 n+2)^{s}}-\frac{1}{(5 n+3)^{s}}+\frac{1}{(5 n+4)^{s}}\right],
$$

and finally another period 8 series

$$
L_{8}(s)=\sum_{n=0}^{\infty}\left[\frac{1}{(8 n+1)^{s}}-\frac{1}{(8 n+3)^{s}}-\frac{1}{(8 n+5)^{s}}+\frac{1}{(8 n+7)^{s}}\right] .
$$

As an example of Nazimov's method, consider the following. He starts with the following relation given by Jacobi [1], p. 101, Equation (18):

$$
\frac{2 K}{\pi}=\operatorname{sn} \frac{2 K x}{\pi}\left[\frac{1}{\sin (x)}+4 \sum_{n=1}^{\infty} \frac{q^{2 n-1}}{1-q^{2 n-1}} \sin (2 n-1) x\right],
$$

where $s n$ is a Jacobian elliptic function. In this equation, Nazimov puts $x=\pi / 4$. Then, it is known that $\operatorname{sn}(K / 2)=1 / \sqrt{ }\left(1+k^{\prime}\right)$. As $n$ advances from 1 to $4,2 n-1$ yields numbers which are $1 \equiv$ $\bmod 8,3 \equiv \bmod 8,5 \equiv \bmod 8,7 \equiv \bmod$ and then repeat themselves. For $(2 n-1)=1,3 \equiv$ $\bmod 8, \sin (2 n-1) \pi / 4=1 \sqrt{ } 2$, whereas for $(2 n-1)=5,7 \equiv \bmod 8, \sin (2 n-1) \pi / 4=-1 \sqrt{ } 2$. Then, rearranging (25), one arrives at

$$
\frac{1}{\sqrt{ } 2} \frac{2 K}{\pi} \sqrt{ }\left(1+k^{\prime}\right)=1+2 \sum_{n=0}^{\infty}\left(\frac{q^{8 n+1}}{1-q^{8 n+1}}+\frac{q^{8 n+3}}{1-q^{8 n+3}}-\frac{q^{8 n+5}}{1-q^{8 n+5}}-\frac{q^{8 n+7}}{1-q^{8 n+7}}\right) .
$$


It is simple to show that the LHS of (26) is equal to $\theta_{3} \theta_{3}\left(q^{2}\right)$. Expanding the denominators of the RHS of (26) then gives

$$
\sum_{-\infty}^{\infty} \sum_{-\infty}^{\infty} q^{m^{2}+2 n^{2}}=1+2 \sum_{m=1}^{\infty} \sum_{n=0}^{\infty}\left[q^{m(8 n+1)}+q^{m(8 n+3)}-q^{m(8 n+5)}-q^{m(8 n+7)}\right]
$$

Nazimov then interprets this result as The number of solutions of the diophantine equation $m^{2}+2 n^{2}=$ $N$ is equal to twice the number of divisors of $N$ of the forms $(8 n+1)$ and $(8 n+3)$ minus twice the number of divisors of $N$ of the forms $(8 n+5)$ and $(8 n+7)$. To yield the equivalent lattice sum, subtract 1 from both sides of (27), replace $q$ by $e^{-t}$ Mellin transform both sides of (27), and one recovers Lorenz's result (10). Nazimov indeed produces the equivalent of Lorenz's results (10)-(13) and several more two-dimensional results, so although they were new when given by Nazimov, now they have not added anything to our knowledge, since in [9] all possible solutions of $\sum^{\prime}\left(a m^{2}+b m n+c n^{2}\right)^{-s}$ capable of being expressed as sums of products of pairs of Dirichlet series were found and given. However, Nazimov goes on to consider many 4, 6, and even a 12 term quadratic forms, all of which can be transformed into lattice sums not previously found, and these will now be given.

As an example of how Nazimov obtains his results, the full details of how he tackled the quadratic form $\left(m^{2}+n^{2}+p^{2}+2 r^{2}\right)$ are now described ([10], p. 17).

Problem 1. To determine the number of solutions of the equation $\left(m^{2}+n^{2}+p^{2}+2 r^{2}\right)=N$. It is necessary in the solution of this problem to find series in powers of $q$ for the expression

$$
\theta_{3}^{3} \theta_{3}\left(q^{2}\right)=\frac{2 K^{2}}{\pi^{2}} \sqrt{ } 2 \sqrt{ }\left(1+k^{\prime}\right)
$$

It is easy to see that

$$
\begin{aligned}
& \frac{4 K^{2}}{\pi^{2}} \sqrt{ } 2 \sqrt{ }\left(1+k^{\prime}\right)=-\frac{4 K^{2}}{\pi^{2}} k c n\left(\frac{K}{2}+K^{\prime} i\right) d n\left(\frac{K}{2}+K^{\prime} i\right) \\
& =\sqrt{ } 2-4 \sum_{n=1}^{\infty} \frac{(2 n-1) q^{2 n-1}}{1-q^{2 n-1}} \cos (2 n-1) \frac{\pi}{4} .
\end{aligned}
$$

As a consequence of this, we obtain

$$
\frac{2 K^{2}}{\pi^{2}} \sqrt{ } 2 \sqrt{ }\left(1+k^{\prime}\right)=1+8 \sum_{1}^{\infty} \frac{n\left(q^{n}-q^{3 n}-q^{5 n}+q^{7 n}\right)}{1-q^{8 n}}-2 \sum_{n=0}^{\infty}(-1)^{\frac{n^{2}+n}{2}} \frac{(2 n+1) q^{2 n+1}}{1-q^{2 n+1}} .
$$

Whether it is "easy" to see (29) or not, the consequences are correct, and in terms of $q$-series, (29) is saying

$$
\begin{aligned}
& \sum_{m, n, p, r=-\infty}^{\infty} q^{m^{2}+n^{2}+p^{2}+2 r^{2}} \\
& =1+8 \sum_{m, n=1}^{\infty} n\left[q^{m(8 n+1)}-q^{m(8 n+3)}-q^{m(8 n+5)}+q^{m(8 n+7)}\right] \\
& -2 \sum_{m, n=1}^{\infty}\left[(8 n-7) q^{m(8 n-7)}-(8 n-5) q^{m(8 n-5)}\right. \\
& \left.\quad-(8 n-3) q^{m(8 n-3)}+(8 n-1) q^{m(8 n-1)}\right] .
\end{aligned}
$$


As before, subtract 1 from both sides of (30), replace $q$ by $e^{-t}$ Mellin transform both sides of (30), and one obtains

$$
\sum^{\prime}\left(m^{2}+n^{2}+p^{2}+2 r^{2}\right)^{-s}=8 \zeta(s-1) L_{8}(s)-2 \zeta(s) L_{8}(s-1) .
$$

This is typical of Nazimov's method of finding the appropriate $q$-series for the chosen quadratic form. Hence, the following four-dimensional lattice sums were extracted from his results. Rather than give the lattice sums found with all the digits displayed, it is more convenient to display the sum as the Mellin transform of the appropriate $\theta$ function combination.

$$
\begin{gathered}
M_{s}\left[\theta_{3}^{2} \theta_{3}^{2}\left(q^{2}\right)-1\right]=4 \eta(s) \zeta(s-1)\left(1+2^{-s}+2^{2-2 s}\right) \\
M_{s}\left[\theta_{3} \theta_{3}^{3}\left(q^{2}\right)-1\right]=4 \zeta(s-1) L_{8}(s)-2 \zeta(s) L_{8}(s-1) \\
\left.M_{s}\left[\theta_{3}^{2} \theta_{3}^{2}\left(q^{3}\right)-1\right]=4\left(1-3^{1-s}\right)\left(1-2^{1-s}+2^{2-2 s}\right) \zeta(s-1) \zeta(s)\right) \\
M_{s}\left[\theta_{3}^{3} \theta_{3}\left(q^{4}\right)-1\right]=4 \eta(s) \zeta(s-1)\left(1+2^{1-s}-2^{-2 s}+2^{2-3 s}\right)+2 \beta(s) \beta(s-1) \\
M_{s}\left[\theta_{3}^{2} \theta_{3}^{2}\left(4^{2}\right)-1\right]=2 \eta(s) \zeta(s-1)\left(1+2^{-s}+2^{1-2 s}+2^{3-3 s}\right)+2 \beta(s) \beta(s-1) \\
M_{s}\left[\theta_{3} \theta_{3}^{3}\left(q^{4}\right)-1\right]=\eta(s) \zeta(s-1)\left(1-2^{-s}+2^{3-2 s}+2^{4-3 s}\right)+\beta(s) \beta(s-1) \\
M_{s}\left[\theta_{3} \theta_{3}^{2}\left(q^{2}\right) \theta_{3}\left(q^{4}\right)-1\right]=2 \eta(s) \zeta(s-1)\left(1+2^{-s}+2^{1-2 s}+2^{3-3 s}\right) \\
\left.M_{s}\left[\theta_{3}^{3} \theta_{3}\left(q^{5}\right)-1\right]=5\left(1+2^{1-s}-2^{2-2 s}\right) \zeta(s-1) L_{5}(s)\right)+\left(1-2^{1-s}-2^{2-2 s}\right) \zeta(s) L_{5}(s-1) \\
\left.M_{s}\left[\theta_{3} \theta_{3}^{3}\left(q^{5}\right)-1\right]=\left(1+2^{1-s}-2^{2-2 s}\right) \zeta(s-1) L_{5}(s)\right)+\left(1-2^{1-s}-2^{2-2 s}\right) \zeta(s) L_{5}(s-1)
\end{gathered}
$$

Of these formulae, (33) and (35)-(37) are easily found from recently found results of lattice sums by use of the relations (4). However, all the other results must be solely ascribed to Nazimov.

Intermingled with the latter's four-dimensional $\theta$ expressions, there are several 6-d relations derived.

$$
\begin{gathered}
3 M_{s}\left[\theta_{3}^{5} \theta_{3}\left(q^{2}\right)-1\right]=32 \zeta(s-2) L_{-8}(s)-2 \zeta(s) L_{-8}(s-2) . \\
M_{s}\left[\theta_{3}^{4} \theta_{3}^{2}\left(q^{2}\right)-1\right]=8 \zeta(s-2) L_{-8}(s)-4.2^{-s} \zeta(s) L_{-8}(s-2) \\
M_{s}\left[\theta_{3}^{5} \theta_{3}\left(q^{3}\right)-1\right]=9\left(1+2^{1-s}-2^{3-2 s}\right) \zeta(s-2) L_{-3}(s)+\left(1-2^{1-s}-2^{3-2 s}\right) \zeta(s) L_{-3}(s-2) . \\
M_{s}\left[\theta_{3} \theta_{3}^{5}\left(q^{3}\right)-1\right]=\left(1+2^{1-s}-2^{3-2 s}\right) \zeta(s-2) L_{-3}(s)+\left(1-2^{1-s}-2^{3-2 s}\right) \zeta(s) L_{-3}(s-2) . \\
M_{s}\left[\theta_{3}^{4} \theta_{4}^{2}-\theta_{3}^{2} \theta_{4}^{4}\right]=8 \lambda(s) \beta(s-2)=\frac{4 \pi^{s-2}}{\Gamma(s)} \int_{0}^{1} \frac{1-k^{\prime}}{k k^{\prime}} K^{2-s}\left(K^{\prime}\right)^{s-1} \mathrm{~d} k . \\
M_{s}\left[\theta_{3}^{6}-\theta_{3}^{2} \theta_{4}^{4}\right]=16 \beta(s) \zeta(s-2)=\frac{4 \pi^{s-2}}{\Gamma(s)} \int_{0}^{1} \frac{k}{1-k^{2}} K^{2-s}\left(K^{\prime}\right)^{s-1} \mathrm{~d} k . \\
M_{s}\left[\theta_{4}^{6}-\theta_{3}^{4} \theta_{4}^{2}\right]=16 \beta(s) \eta(s-2)=\frac{4 \pi^{s-2}}{\Gamma(s)} \int_{0}^{1} \frac{k}{k^{\prime}} K^{2-s}\left(K^{\prime}\right)^{s-1} \mathrm{~d} k .
\end{gathered}
$$

In the last three equations, it will be seen that extra information has been adduced from another source. The origin of this is a recent paper [11], which showed that any lattice sum involving $\theta$-functions 
all having the same argument could be transformed in a simple manner into integrals of combinations of $k, k^{\prime}, K, K^{\prime}$. In particular, (47) yield for $s=1,2,3$, respectively, the following neat results:

$$
\int_{0}^{1} \frac{k}{k^{\prime}} K \mathrm{~d} k=\frac{\pi^{2}}{4}, \quad \int_{0}^{1} \frac{k}{k^{\prime}} K^{\prime} \mathrm{d} k=2 G, \quad \int_{0}^{1} \frac{k}{k^{\prime}} \frac{K^{\prime 2}}{K} \mathrm{~d} k=\frac{\pi^{2} \log 2}{4} .
$$

In the above expressions, $G$ is Catalan's constant and is equal to $\beta(2)$.

Finally, Nazimov produced a result involving 12-dimensional lattice sums

$$
M_{s}\left[\theta_{2}^{8} \theta_{3}^{4}+\theta_{2}^{4} \theta_{3}^{8}\right]=16 \lambda(s) \zeta(s-5)=\frac{32 \pi^{s-5}}{\Gamma(s)} \int_{0}^{1} \frac{k\left(1+k^{2}\right)}{k^{\prime 2}} K^{5-s}\left(K^{\prime}\right)^{s-1} \mathrm{~d} k .
$$

Remarkably, this is not unique, as in a completely different manner [10] produced the following result:

$$
M_{s}\left[2 \theta_{2}^{12}-3 \theta_{2}^{8} \theta_{3}^{4}+\theta_{2}^{4} \theta_{3}^{8}\right]=16 \lambda(s) \eta(s-5)=\frac{32 \pi^{s-5}}{\Gamma(s)} \int_{0}^{1} k\left(1-2 k^{2}\right) K^{5-s}\left(K^{\prime}\right)^{s-1} \mathrm{~d} k .
$$

Unfortunately, this does not allow us to identify a single 12-dimensional sum on its own.

It should be pointed out that all the lattice sums derived here from Nazimov's work have been obtained from the first 40 pages of his 146-page pamphlet, most of which is dedicated to finding arithmetical properties of algebraic forms. It is not claimed that all possible lattice sums have been retrieved from Nazimov. His booklet is certainly worthy of further study.

Acknowledgments: I am most grateful to my colleague M. L. Glasser for discovering Nazimov's booklet and passing it on to me.

Conflicts of Interest: The author declares no conflict of interest.

\section{References}

1. Jacobi, C.G. Fundamenta Nova Theoriae Funcionum Ellipticarum; Sumtibus Fratrum Borntraeger: Königsberg, Russia, 1829.

2. Hardy, G.H. On some integral considered by Mellin. Messenger Math. 1919, 49, 85-91.

3. Lorenz, L. Bidrag til talenes theori. Tidsskr. Fur. Math. 1871, 1, 97-114.

4. Madelung, E. Das elektrische Feld in Systemen von regelmaßig angeordnetenPunktladungen. Phys. Z. 1918, 19, 524-533.

5. Glasser, M.L. The evaluation of lattice sums. I. Analytic procedures. J. Math. Phys. 1973, 14, 409-413.

6. Glasser, M.L. The evaluation of lattice sums. II. Number-theoretic approach. J. Math. Phys. 1973, 14, 701-703.

7. Zucker, I.J. A note on lattice sums in two dimensions. J. Math. Phys. 1974, 15, 187.

8. Glasser, M.L.; Zucker, I.J. Lattice Sums. In Theoretical Chemistry, Advances and Perspectives; Academic Press: New York, NY, USA, 1980; Volume 5, pp. 67-139, ISBN 0-12-681905.

9. Borwein, J.M.; Glasser, M.L.; McPhedran, R.C.; Wan, J.G.; Zucker, I.J. Lattice Sums then and now. In Encyclopedia of Mathematics and Its Applications; C.U.P.: Cambridge, UK, 2013; ISBN 978-1-107-03990-2.

10. Nazimov, P.S. Applications of the Theory of Elliptic Functions to the Theory of Numbers; University of Chicago Bookstore: Chicago, IL, USA, 1928.

11. Wan, J.G.; Zucker, I.J. Integrals of $K$ and $E$ from lattice sums. Raman. J. 2016, 40, 257-278.

(C) 2017 by the author. Licensee MDPI, Basel, Switzerland. This article is an open access article distributed under the terms and conditions of the Creative Commons Attribution (CC BY) license (http:// creativecommons.org/licenses/by/4.0/). 